\title{
Los hábitos de actividad física de las mujeres mayores en España. The habits of physical activity of the older women in Spain.
}

\author{
Jesús Martínez del Castillo(1); María Dolores González Rivera (2); \\ José Emilio Jiménez-Beatty Navarro(2); José Luis Graupera Sanz (2); \\ María Martín Rodríguez(1); Antonio Campos Izquierdo(1); David Del Hierro Pinés(1)
}

(1) Universidad Politécnica de Madrid

(2)Universidad de Alcalá de Henares

\section{Resumen}

Dada la inexistencia de estudios precedentes de hábitos de actividad física en el conjunto de mujeres mayores de 65 años en España, la investigación persigue comprobar las posibles desigualdades y diferencias en la práctica de actividad física semanal de las mujeres mayores con respecto a los hombres. La metodología cuantitativa empleada ha consistido en la aplicación de un cuestionario estructurado a una muestra aleatoria de las mujeres y varones mayores de 65 años en España. Las conclusiones obtenidas revelan que en este grupo sociodemográfico de las personas mayores de 65 años, y a diferencia de los otros grupos con menor edad, un mayor porcentaje de mujeres que de varones realiza actividad física semanal (sin incluir el pasear). Esta desigualdad viene acompañada y quizás explicada por una serie de diferencias en la relación con la práctica de actividad física por parte de las mujeres mayores en comparación a gran parte de los hombres mayores, y que quizás configuren lo que podría denominarse como una cultura deportiva femenina de las mujeres mayores: tanto en las actividades practicadas, como en el espacio utilizado, el modo de organización de la actividad, y en el modo de acudir a la actividad.

Palabras clave: hábitos; actividad física; mujeres mayores.

\section{Abstract}

Given the absence of previous studies of habits of physical activity in the set of 65 year older women in Spain, the investigation claims to verify the possible inequality and differences in the practice of physical weekly activity of the older women with regard to the men. The quantitative methodology has consisted of the application of a questionnaire constructed to a random sample of the 65 year older persons of Spain. The conclusions reveal that in this demographic group of the older, and unlike other groups with minor age, a major percentage of women that of males it realizes physical weekly activity (without including to walk). This inequality comes accompanied and probably explained by a series of differences in the relation with the practice of physical activity on the part of the older women in comparison to great part of the older men, and that probably form what might be named as a sports feminine culture of the older women: in the practiced activities, in the used space, in the way of organization of the activity, like in the way of coming to the activity.

Key words: habits; physical activity; older women. 
Martínez, J.; Jiménez-Beatty J.E.; González, M.D.; Graupera, J.L.; Martín, M.; Campos, A.; Del Hierro, D. (2009). Los hábitos de actividad física de las mujeres mayores en España. Revista Internacional de Ciencias del Deporte. 14(5), 81-93. http://www.cafyd.com/REVISTA/01407.pdf

\section{Introducción}

$\mathrm{E}$ n las investigaciones de hábitos deportivos en España ha sido habitual hasta hace pocos años, estudiar a la población sólo hasta los 65 años. Así se hizo en el estudio pionero sobre hábitos deportivos y mujer de Vázquez (1993) o en todos los estudios efectuados por García Ferrando hasta 1995 inclusive. Es en el estudio de hábitos deportivos de 2000, cuando García Ferrando (2001) incorpora al universo y la muestra personas de 65 a 74 años, encontrando que practicaban actividad física el $8 \%$, de los cuales el $54 \%$ lo hacía tres veces a la semana o más. Y en el último estudio de 2005, García Ferrando (2006) midió con respecto a las personas de 65 a 74 años, que practicaban actividad física el $17 \%$, de los cuales el $56 \%$ lo hacía tres veces a la semana o más. A partir de estos resultados y de los obtenidos entre los practicantes mayores de 55 años, García Ferrando (2006) señala un manifiesto incremento de frecuencia de actividad entre los practicantes mayores de 55 años, considerando este incremento como un cambio significativo que habrá que seguir en próximas encuestas.

En cuanto a los hábitos deportivos de los españoles (varones y mujeres) de 15 a 74 años, García Ferrando (2006) obtuvo entre otros los siguientes resultados: el 37\% practicaba uno o más deportes; en la frecuencia de práctica, el $49 \%$ practicaba tres veces o más por semana, el $37 \%$ una o dos veces por semana, el 10\% con menor frecuencia y el 3\% sólo en vacaciones; los deportes (entendidos en su significado más amplio, es decir incluyendo actividades físicas no deportivas) más practicados en orden decreciente son la Natación (por el 33\%), el Fútbol (31,7\%), el Ciclismo (19,1\%), la Gimnasia de mantenimiento en centro deportivo, (14\%), el Montañismo (11,9\%), las Actividades de Aeróbic y similares (el 11,7\%), la carrera a pie $(11,1 \%)$ (el resto de deportes y actividades aparecen con frecuencias inferiores al $10 \%)$; en la forma de hacer deporte, el $68 \%$ lo hace por su cuenta, el $24 \%$ en alguna organización (no incluida entre las siguientes), el $6 \%$ en el centro de enseñanza o de trabajo en el que está; las instalaciones utilizadas eran instalaciones públicas por un 51\%, lugares públicos por un $43 \%$, instalaciones de un club privado por un $20 \%$, en un gimnasio privado por un $8 \%$, en la propia casa por el $1 \%$.

Respecto a los hábitos deportivos de las mujeres de 15 a 74 años, García Ferrando (2006) muestra los siguientes resultados: el 30\% practicaba uno o más deportes (frente al $45 \%$ de los varones); el $49 \%$ practica tres veces o más por semana (igual que los varones); el $24 \%$ practica en alguna organización (frente al 23\% de los varones); en las instalaciones o espacios, un $48 \%$ de las mujeres acude a instalaciones públicas (frente al $54 \%$ de los varones), un $21 \%$ en instalaciones privadas (frente al $19 \%$ de los varones) y un $34 \%$ en lugares abiertos (frente al $49 \%$ de los varones).

Y en referencia a las personas (varones y mujeres) de 65 a 74 años, los hábitos deportivos eran (García Ferrando, 2006): el 17\% practicaba uno o más deportes; el 56\% practica tres veces o más por semana (siendo el grupo sociodemográfico que practica más frecuentemente); en las instalaciones o espacios, un $42 \%$ acude a instalaciones públicas (frente al 61\% de las personas de 15 a 24 años), un 13\% en instalaciones privadas (frente al $19 \%$ de las personas de 15 a 24 años) y un $46 \%$ en lugares abiertos (frente al $34 \%$ de las personas de 15 a 24 años).

Sin embargo los datos de hábitos deportivos específicos de las mujeres de 65 a 74 años en España no aparecen reseñados en los dos citados estudios de García Ferrando de 2001 y 2006.

Con respecto a los estudios y teorías sobre género y deporte, García Ferrando $(2001,2006)$ así como Mosquera y Puig (2002), y Puig y Soler (2004), han señalado a partir de la clara 
Martínez, J.; Jiménez-Beatty J.E.; González, M.D.; Graupera, J.L.; Martín, M.; Campos, A.; Del Hierro, D. (2009). Los hábitos de actividad física de las mujeres mayores en España. Revista Internacional de Ciencias del Deporte. 14(5), 81-93. http://www.cafyd.com/REVISTA/01407.pdf

evidencia empírica de los estudios de hábitos deportivos en España, las desigualdades en el acceso a la práctica del conjunto de las mujeres de 15 a 74 años frente al conjunto de los varones de 15 a 74 años y las diferencias entre hombres y mujeres en diferentes variables (entre otras, en las actividades practicadas, en los motivos, en la frecuencia, en la entidad en la que practican...). Todo ello lleva a García Ferrando $(2001,2006)$ a señalar dos formas diferentes de relacionarse con el deporte. Incluso Puig y Soler (2004) van más allá y plantean con respecto al conjunto de las mujeres de todas las edades, que a medida que las españolas han ido accediendo al deporte, lejos de reproducir comportamientos masculinos, lo han modelado según otros parámetros, han creado una cultura deportiva propia: una cultura deportiva femenina. Según Puig y Soler (2004) las propuestas teóricas de análisis de Martín (1999) a partir de los planteamientos de Bourdieu, de considerar que las personas que practican deporte configuran un "сатро” con interacciones y diversidades generados por los capitales (económico, cultural, social y simbólico) de que son portadoras, contribuyen a explicar que las mujeres configuren "campos deportivos" de modo distinto al de los hombres. Asimismo Puig y Soler señalan que las organizaciones en las que parecen practicar más las mujeres que los hombres, son los gimnasios privados, pero en los clubes deportivos (orientados a la competición), se da la tendencia contraria. Ambas tendencias podrían estar relacionadas con las culturas organizativas predominantes y los planteamientos de la oferta de esas organizaciones, al estar más en sincronía con las mujeres el sector privado comercial, que el sector asociativo tradicional. En los casos del sector público municipal, si bien no hay datos estadísticos generales, Puig y Soler observan que en los casos que se ha registrado una adecuada adaptación a las mujeres, gran parte de éstas (y entre ellas, aquellas que por sus posibilidades económicas no podían acceder al sector privado comercial) se han incorporado a los diferentes programas municipales de promoción.

Además Puig y Soler (2004) sugieren abordar las diferencias en las prácticas de las mujeres y las diferentes desigualdades en el deporte (como el menor acceso a la práctica en el conjunto de las mujeres frente a los hombres) desde dos perspectivas teóricas: la del feminismo de la diferencia y la de la desigualdad. Para ambas autoras la incorporación de la mujer al mundo del deporte, ha ido en paralelo a la construcción y actuaciones del Estado del Bienestar, y a la diversificación de organizaciones y modos de entender y practicar deporte. Y dentro de esos procesos, las mujeres han construido y reproducido su identidad de género de modo distinto a los hombres y una cultura deportiva femenina. Pero ello "no significa ni mucho menos que las mujeres se limiten a reproducir el estereotipo hegemónico de feminidad consolidando así situaciones de desigualdad-y hasta de opresión- frente a los hombres. Es, por el contrario, proyectar en la sociedad valores y modos de hacer más acordes con los deseos y necesidades de las mujeres.”(Puig y Soler, 2004, 76).

En ámbitos territoriales más reducidos, sí se han hecho estudios y se dispone de datos específicos de los hábitos de actividad física de las mujeres mayores de 65 años. Así en el estudio de Graupera, Martínez del Castillo y Martín (2003) sobre una muestra de mujeres mayores (con una edad media de 69.3 años) participantes en programas deportivos municipales en la Comunidad de Madrid se observaron los siguientes hábitos: la totalidad de estas mujeres realizaba actividades de Gimnasia de Mantenimiento en polideportivos u otras instalaciones de sus municipios de residencia, organizadas por los respectivos ayuntamientos, como miembros de un grupo, supervisado o dirigido por un profesor; en el 79\% de los casos, dos sesiones a la semana y en el 19\%, tres veces, y siempre entre semana por las mañanas; a dicha actividad acudían andando la mayoría, (62\%); y un 22\% en autobús; sólo el $12 \%$ empleaba el coche. 
Martínez, J.; Jiménez-Beatty J.E.; González, M.D.; Graupera, J.L.; Martín, M.; Campos, A.; Del Hierro, D. (2009). Los hábitos de actividad física de las mujeres mayores en España. Revista Internacional de Ciencias del Deporte. 14(5), 81-93. http://www.cafyd.com/REVISTA/01407.pdf

En el estudio de Jiménez-Beatty, Graupera y Martínez del Castillo (2003) en una muestra aleatoria de los varones y mujeres mayores de 65 años del municipio de Madrid, se encontró que realizaban actividad física el 17,9\% de las mujeres (y el 17,3\% de los hombres). Con respecto a las actividades físicas efectuadas (sin incluir el pasear, que lo hacía casi la totalidad): en los casos de las mujeres, la mayoría (un 67\%) realizaban gimnasia de mantenimiento o actividad similar, un 16\% practican natación (o más precisamente alguna actividad física en el medio acuático) y en menor medida el yoga, un $9 \%$ u otras actividades como el baile, el aeróbic o la petanca; en los varones era la natación la principal actividad (31\%), seguida de la gimnasia de mantenimiento, un $21 \%$, y en menor frecuencia la petanca y la bicicleta con un $10 \%$ cada uno, el tenis (7\%) u otras actividades. Respecto a los hábitos y características de su actividad: estas mujeres practicaban con gran regularidad, dos veces a la semana o más, y en mayor medida por las mañanas; su práctica solía estar organizada por alguna entidad y realizada en un grupo dirigido por un profesor; y suelen realizar la actividad en un espacio deportivo, al que la mayoría, acuden andando.

Más recientemente, el estudio de Jiménez-Beatty, Martínez del Castillo y Graupera (2006) en una muestra aleatoria de los varones y mujeres mayores de 65 años de la provincia de Guadalajara, reveló que realizaban actividad física (sin incluir el pasear, que lo hacía la mayoría) el 18,8\% de las personas mayores: el 23,6\% de las mujeres (y el 13,6\% de los hombres). En lo que respecta a las actividades practicadas por el conjunto de personas mayores se obtuvieron: la gimnasia de mantenimiento (más de la mitad), y a más distancia: pasear en bicicleta, la natación, el baile, los deportes de raqueta, la petanca, los bolos, el yoga y la caza. Las mujeres sobre todo practican la gimnasia de mantenimiento (73\%), y los hombres por su parte diversifican más sus actividades, siendo las principales: la gimnasia de mantenimiento (25,5\%), la bicicleta (24\%) y la caza (12\%). La frecuencia de práctica es de dos días a la semana o más en el $88 \%$ de las mujeres y en el $78 \%$ de los varones. Acuden a practicar bien por las mañanas (48\%) o bien por las tardes (41\%): los varones acuden más por las mañanas, y las mujeres acuden más a diario por las tardes. Practican en entidades públicas o privadas (un 60\%), sobre todo las mujeres, pues los hombres se autoorganizan más (50\%). El 63\% practica con presencia de técnico, sobre todo mujeres; por el contrario, tres de cada cuatro hombres practican sin presencia de profesor. Practican en instalaciones deportivas (18\%), sobre todo las mujeres (más del doble) o en centros con instalación (el 27,5\%), pero un $40 \%$ de los hombres practican en parques, plazas u otros espacios simbólicos. Y la mayoría acuden andando a la instalación, especialmente las mujeres.

Pues bien a fin de profundizar en el conocimiento del posible cambio significativo en la práctica de las personas mayores pronosticado por García Ferrando, y dado el vacío existente sobre los hábitos deportivos específicos de las mujeres mayores en España, los objetivos del

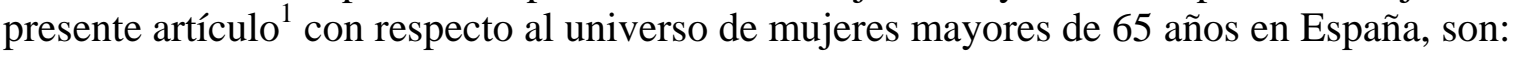

o Establecer las tasas de práctica de actividad física semanal de las mujeres mayores y comprobar si hay desigualdad con los varones mayores.

o Identificar los hábitos de actividad física de las mujeres mayores y comprobar si hay diferencias con los varones mayores.

\section{Material y método}

La metodología ha consistido en la realización de entrevistas estructuradas a una muestra estadísticamente representativa de las mujeres y hombres de 65 años cumplidos o más, en 
Martínez, J.; Jiménez-Beatty J.E.; González, M.D.; Graupera, J.L.; Martín, M.; Campos, A.; Del Hierro, D. (2009). Los hábitos de actividad física de las mujeres mayores en España. Revista Internacional de Ciencias del Deporte. 14(5), 81-93. http://www.cafyd.com/REVISTA/01407.pdf

España (4.319.863 mujeres y 3.164.529 hombres, según el INE y el Padrón Municipal a 1-12006).

Dicha muestra registra las siguientes características: el tamaño de la muestra final ha sido de 933 personas mayores, de las que el 53,1 \% son mujeres y el 46,9 \% varones; dado que es una población infinita o muy numerosa, y trabajando con un intervalo de confianza del 95,5\%, y suponiendo en la varianza poblacional el caso más desfavorable de $\mathrm{p}$ igual a 50, luego q = 50 , el margen de error permitido de muestreo es de $\pm 3,27 \%$. El tipo de muestreo, ha sido probabilístico de tipo polietápico, siendo las unidades de primera etapa los municipios en que residían habitualmente las personas mayores. La afijación de la muestra, ha sido proporcional a la distribución de las personas mayores según tamaño demográfico de los municipios y según género.

El instrumento empleado para captar la información necesaria para los objetivos del estudio, ha sido elaborado a partir del "Cuestionario de Actividad Física y Personas Mayores" elaborado por Graupera Sanz y Martínez del Castillo para medir los hábitos de actividad física de las personas mayores y otras variables relacionadas (Graupera et al., 2003). Dicho cuestionario ya ha sido validado en anteriores estudios sobre personas mayores (JiménezBeatty et al., 2003; Jiménez-Beatty et al., 2007).

El trabajo de campo se llevó a cabo durante el mes de diciembre de 2006. En él, los entrevistadores aplicaron el cuestionario escrito mediante entrevista personal estructurada cara a cara, en el domicilio habitual del mayor. Los análisis de datos fueron efectuados, tras ser tabulados y mecanizados informáticamente, mediante el paquete informático de programas SPSS para WINDOWS (versión 14.0).

\section{Resultados}

Con respecto a la realización semanal de actividad física o deportiva (sin incluir el pasear), el $19,6 \%$ de las mujeres mayores declaran practicar semanalmente una o más actividades físicas o deportivas, frente al 14,8\% de los hombres mayores. Sin embargo en los hábitos de pasear se ha obtenido un porcentaje mayor de varones que salen a caminar que de mujeres $(92,2 \%$ frente a un 81,9\%).

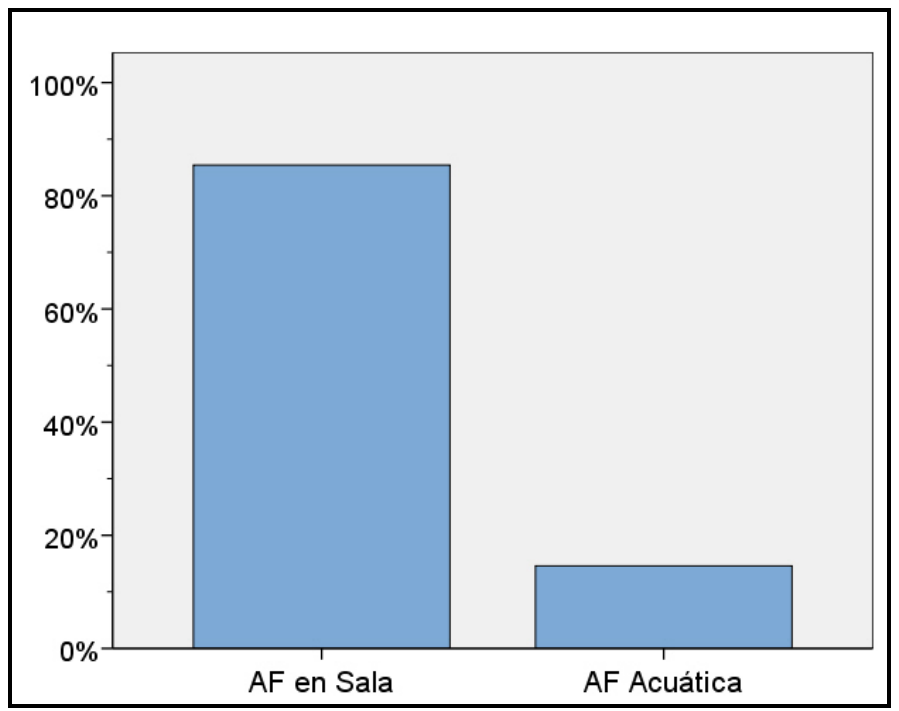

Figura 1. Categorías de actividades físicas practicadas por las mujeres 
Martínez, J.; Jiménez-Beatty J.E.; González, M.D.; Graupera, J.L.; Martín, M.; Campos, A.; Del Hierro, D. (2009). Los hábitos de actividad física de las mujeres mayores en España. Revista Internacional de Ciencias del Deporte. 14(5), 81-93. http://www.cafyd.com/REVISTA/01407.pdf

En las actividades practicadas las mujeres mayores (Figura 1) concentran sus actividades físicas en espacios cubiertos (el 85,4\% Actividades Físicas en Sala, y el otro 14,6\% Actividades Físicas Acuáticas), pero en los hombres (Figura 2) se diversifican algo más los tipos de actividad y de espacio: un elevado 67\% realiza AF en Sala, y un 12,5\% Actividades Físicas Acuáticas; pero hay otros varones mayores en los que su principal actividad semanal son las AF al aire libre, para el 17\% (Juegos de lanzamiento - petanca, bolos en parques, el 3,1\%; desplazamientos con la bicicleta en medio urbano, el 3,1\%; y Actividades en la Naturaleza - senderismo, pesca, caza-, el 10,9\%) y un 3,1\% practica algún Deporte. A estas diferencias se añaden otras en algunas de las Actividades Física en Sala, pues la AF y música - bailes-, es practicada por un porcentaje similar de mujeres y hombres, el 3,1\%, pero en las mujeres hay un mayor porcentaje de practicantes de programas de ejercicio físico (el 69,8\% frente al 60,9\% de los varones) y de actividades de gimnasia oriental - taichi, yoga- (el 12,5\% frente al 3,1\% de los varones.)

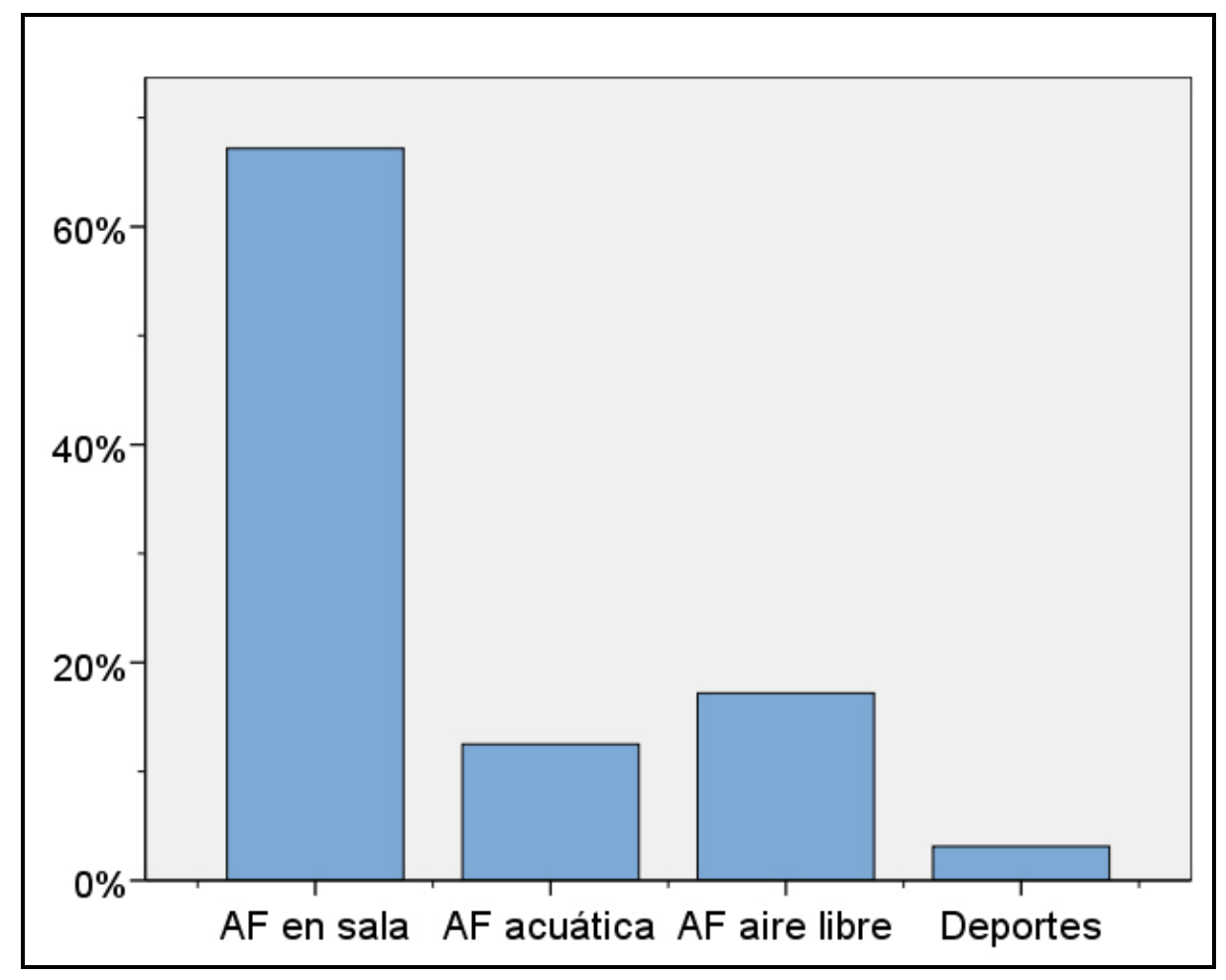

Figura 2. Categorías de actividades físicas practicadas por los varones

En cuanto a las horas de práctica semanal (Tabla 1), prácticamente todas las mujeres (el $96,9 \%$ ), realizan dos horas o más a la semana con una media de 2.53 horas. Sin embargo, en los varones hay un 17,5\% que practica sólo una hora a la semana, pero el resto es decir el 82,5\% realiza dos horas o más a la semana, siendo la media de todos los varones de 3.46 horas (casi una hora más a la semana que las mujeres, probablemente porque parte de los varones realizan actividades al aire libre y en la naturaleza que implican más tiempo de práctica). 
Martínez, J.; Jiménez-Beatty J.E.; González, M.D.; Graupera, J.L.; Martín, M.; Campos, A.; Del Hierro, D. (2009). Los hábitos de actividad física de las mujeres mayores en España. Revista Internacional de Ciencias del Deporte. 14(5), 81-93. http://www.cafyd.com/REVISTA/01407.pdf

Tabla 1. Horas semanales de práctica

\begin{tabular}{|c|r|r|r|}
\hline Horas de práctica & Hombre & \multicolumn{1}{c|}{ Mujer } & \multicolumn{1}{c|}{ Total } \\
\hline 1 & $17.5 \%$ & $3.1 \%$ & $8.8 \%$ \\
\hline 2 & $25.4 \%$ & $54.2 \%$ & $42.8 \%$ \\
\hline 3 & $27.0 \%$ & $32.3 \%$ & $30.2 \%$ \\
\hline$>3$ & $30.2 \%$ & $10.4 \%$ & $18.2 \%$ \\
\hline Promedio & 3.46 & 2.53 & 2.9 \\
\hline Desv. típica & 2.71 & 0.87 & 1.89 \\
\hline
\end{tabular}

El análisis de la localización temporal (Tabla 2) revela que las mujeres van más sólo por las mañanas, el 75\%, que los hombres, el 57,8\%. Mientras que en las personas que van sólo por las tardes, no hay diferencias entre mujeres y hombres, en torno al 19\%. Ahora bien se observa un mayor porcentaje de hombres que van por la mañana o por la tarde, un 21,9\% frente a un $6,3 \%$ de las mujeres.

Tabla 2. Localización temporal de la práctica según género

\begin{tabular}{|l|r|r|r|}
\hline Cuándo practica & Hombre & \multicolumn{1}{c|}{ Mujer } & \multicolumn{1}{c|}{ Total } \\
\hline Mañana & $57.8 \%$ & $75.0 \%$ & $68.1 \%$ \\
\hline Tarde & $20.3 \%$ & $18.8 \%$ & $19.4 \%$ \\
\hline Mañana y tarde & $21.9 \%$ & $6.3 \%$ & $12.5 \%$ \\
\hline
\end{tabular}

La mayoría de las personas mayores que practican (el 72\%), lo hacen a través de alguna organización (Figura 3) situándose en orden decreciente de la siguiente forma: en Centros u Hogares de la $3^{a}$ Edad (25.8\%), en Ayuntamientos (un 22.6\% de los casos), en Gimnasios o Empresas privadas (14,5\%), y en menor medida en Asociaciones (8.2\% respectivamente). En cuanto al resto, el 27.8\% autoorganizaba su actividad, bien sólo por su cuenta (el 20.5\%) o con amigos o familiares (el 7.3\%).

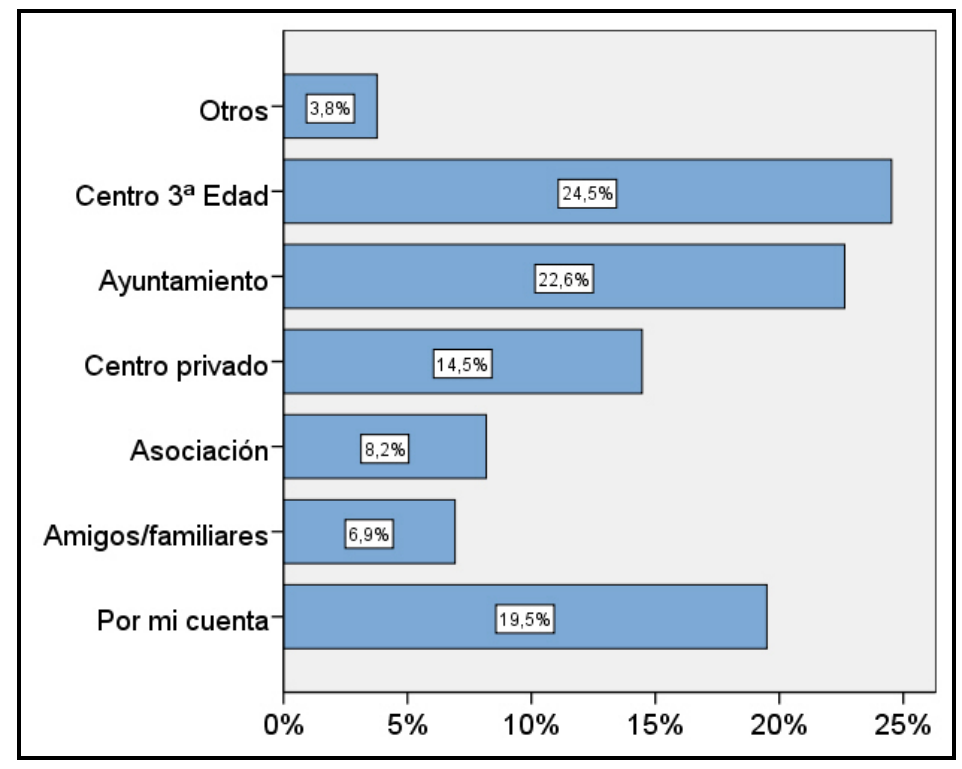

Figura 3. Modo de organización de la actividad física realizada por las mujeres y hombres mayores 
Martínez, J.; Jiménez-Beatty J.E.; González, M.D.; Graupera, J.L.; Martín, M.; Campos, A.; Del Hierro, D. (2009). Los hábitos de actividad física de las mujeres mayores en España. Revista Internacional de Ciencias del Deporte. 14(5), 81-93. http://www.cafyd.com/REVISTA/01407.pdf

Ahora bien la distribución de esta variable modo de organización según el género, revela notables diferencias (tabla 3): el 51,5\% de los hombres auto-organiza su actividad (casi siempre por su cuenta), mientras que sólo el 9,5\% de las mujeres auto-organiza su actividad, es decir que la mayoría recurre a alguna entidad para practicar. Asimismo en el tipo de entidad utilizada se observa que las mujeres van sobre todo a Centros de Mayores (34,7\%) y de Ayuntamientos (28,4\%), seguidos por Empresas Privadas (13,7\%) y Asociaciones (9,5\%). En cambio los varones utilizan los servicios de Empresas Privadas (15,6\%), Ayuntamientos $(14,1 \%)$, Centros de Mayores (9,4\%) y Asociaciones (6,3\%).

Tabla 3. Modo de organización de la actividad física realizada según género

\begin{tabular}{|l|r|r|r|}
\hline Modo de organización & Hombre & Mujer & \multicolumn{1}{c|}{ Total } \\
\hline Por mi cuenta & $40.6 \%$ & $5.3 \%$ & $19.5 \%$ \\
\hline Amigos/familiares & $10.9 \%$ & $4.2 \%$ & $6.9 \%$ \\
\hline Asociación & $6.3 \%$ & $9.5 \%$ & $8.2 \%$ \\
\hline Centro privado & $15.6 \%$ & $13.7 \%$ & $14.5 \%$ \\
\hline Ayuntamiento & $14.1 \%$ & $28.4 \%$ & $22.6 \%$ \\
\hline Centro 3a Edad & $9.4 \%$ & $34.7 \%$ & $24.5 \%$ \\
\hline Otros & $3.1 \%$ & $4.2 \%$ & $3.8 \%$ \\
\hline
\end{tabular}

En referencia a la presencia de técnico durante la realización de la actividad, las mujeres practican mayoritariamente con presencia de técnico (un 81,3\%), mientras que sólo un tercio de los hombres practican con presencia de profesor (un 37,5\%). Es decir que mientras que sólo un 18,8\% de las mujeres practican su actividad sin la presencia de un técnico, en los varones esta situación se da en más de la mitad de los casos, en el 62,5\%.

Asimismo en las instalaciones o espacios utilizados, hay unos perfiles diferenciados (tabla 4). Las mujeres no practican en el propio hogar (sólo el 3,1\%) o en parques o lugares públicos. Casi todas las mujeres realizan su actividad física en instalaciones o centros con espacios de actividad física: el 39,6\% en instalaciones deportivas; el 38,5\% en Centros de Mayores; el $15,6 \%$ en Centros con instalación deportiva. Sin embargo los hombres practican en gran número (el 42\%) en el propio hogar o en parques y plazas. Sólo el $45 \%$ de los varones realiza su actividad en instalaciones o centros con espacios de actividad física: el $29,7 \%$ en instalación deportiva; el 9,4\% en Centros de Mayores; el 6,3\% en Centros con instalación deportiva.

Tabla 4. Instalaciones o espacios utilizados para la práctica según género

\begin{tabular}{|l|r|r|r|}
\hline Espacio de práctica & Hombre & \multicolumn{1}{c|}{ Mujer } & \multicolumn{1}{c|}{ Total } \\
\hline Hogar & $31.3 \%$ & $3.1 \%$ & $14.4 \%$ \\
\hline Parque/plaza & $10.9 \%$ & $0.0 \%$ & $4.4 \%$ \\
\hline Instalación deportiva & $29.7 \%$ & $39.6 \%$ & $35.6 \%$ \\
\hline Centro con inst. dep. & $6.3 \%$ & $15.6 \%$ & $11.9 \%$ \\
\hline Centro 3a Edad & $9.4 \%$ & $38.5 \%$ & $26.9 \%$ \\
\hline Otros & $12.5 \%$ & $3.1 \%$ & $6.9 \%$ \\
\hline
\end{tabular}


Martínez, J.; Jiménez-Beatty J.E.; González, M.D.; Graupera, J.L.; Martín, M.; Campos, A.; Del Hierro, D. (2009). Los hábitos de actividad física de las mujeres mayores en España. Revista Internacional de Ciencias del Deporte. 14(5), 81-93. http://www.cafyd.com/REVISTA/01407.pdf

En las posibles variaciones en el modo de desplazamiento al lugar de práctica según el género, las mujeres acuden prácticamente todas andando $(84,8 \%)$ y muy pocas usan el coche o el autobús. Sin embargo sólo la mitad de los hombres van caminando (55,6\%) y un 25,9\% emplean el coche u otros medios (16,7\%).

El tiempo de desplazamiento al lugar donde realizan la actividad física semanal se sitúa por debajo de los 10 minutos para la mayoría de los practicantes (la media es de 8,7 minutos) (tabla 5): la mitad emplea menos de 5 minutos (el 54,7\%) y la cuarta parte tarda entre 6 y 10 minutos (24,4\%). Tan sólo el 13,8\% emplea entre 11 y 15 minutos, y el 11,3\% más de 15 minutos. Sin embargo en esta variable no hay apenas diferencias según el género y los promedios de mujeres y hombres son similares.

Tabla 5. Tiempo de desplazamiento al lugar de práctica según género

\begin{tabular}{|l|r|r|r|}
\hline $\begin{array}{l}\text { Tiempo de } \\
\text { desplazamiento(minutos) }\end{array}$ & Hombre & Mujer & Total \\
\hline $0-5$ & $54.7 \%$ & $47.9 \%$ & $50.6 \%$ \\
\hline $6-10$ & $14.1 \%$ & $31.3 \%$ & $24.4 \%$ \\
\hline $11-15$ & $20.3 \%$ & $9.4 \%$ & $13.8 \%$ \\
\hline$>15$ & $10.9 \%$ & $11.5 \%$ & $11.3 \%$ \\
\hline Promedio & 8.33 & 8.99 & 8.73 \\
\hline Desviación típica & 9.31 & 6.20 & 7.58 \\
\hline
\end{tabular}

\section{Discusión}

Con respecto a la realización semanal de actividad física o deportiva (sin incluir el pasear), se ha observado en el presente estudio que el $19,6 \%$ de las mujeres declaran practicar semanalmente una o más actividades físicas o deportivas, frente al 14,8\% de los hombres. Es decir que hay desigualdad pero en este segmento sociodemográfico de las personas mayores de 65 años es a favor de las mujeres pues practican actividad física semanalmente (sin incluir el pasear) en un mayor porcentaje que los hombres. Este resultado diverge con los resultados mostrados hasta ahora en los hábitos deportivos del conjunto de españoles y españolas (Vázquez, 1993; García Ferrando, 2001, 2006) y las conclusiones efectuadas en torno a la mayor práctica de los varones frente a las mujeres. Ahora bien en estudios específicos sobre muestras de personas mayores sí parece haber coincidencia en la tendencia hallada en la presente investigación. Así en el estudio efectuado en el municipio de Madrid (JiménezBeatty et al., 2003) ya se observó igualdad entre ambos géneros. E incluso años más tarde en el estudio realizado en la provincia de Guadalajara (Jiménez-Beatty et al., 2006) ya se encontró desigualdad a favor de las mujeres mayores, en una proporción incluso superior a la del presente estudio.

Así pues y a la espera de nuevos estudios, todo parece indicar que si bien en las primeras etapas del ciclo de vida hay desigualdad en las tasas de práctica a favor de los varones, o en el conjunto de las mujeres comparadas con los hombres, al llegar a la vejez hay un número significativo de mujeres (mayor que en los varones) que incorporan la actividad física semanal a sus estilos de vida. Ello podría deberse, siguiendo a Mosquera y Puig (2002) y Martínez del Castillo et al. (2006), a que al producirse una serie de cambios en las circunstancias de vida y entornos de las personas mayores, éstas van incorporando la actividad física cuando les es posible. Es decir, tal como se avanzaba en un estudio anterior 
Martínez, J.; Jiménez-Beatty J.E.; González, M.D.; Graupera, J.L.; Martín, M.; Campos, A.; Del Hierro, D. (2009). Los hábitos de actividad física de las mujeres mayores en España. Revista Internacional de Ciencias del Deporte. 14(5), 81-93. http://www.cafyd.com/REVISTA/01407.pdf

(Martínez del Castillo et al., 2006), si bien los procesos de socialización en la actividad física durante el ciclo de vida aumentan las probabilidades de practicar en la vejez, no determinan dicha práctica, pues los nuevos entornos de socialización durante la vejez pueden impulsar a la práctica a mujeres mayores anteriormente sedentarias. Pues bien este significativo cambio social y deportivo, de desigualdad a favor de las mujeres, puede deberse también, de manera interrelacionada con los procesos anteriormente citados, a una cultura deportiva femenina diferente a la de los hombres, expresada a través de las diferencias en el modo en que se plantean y realizan la actividad física las mujeres mayores, y que a continuación se abordan.

Entre esas diferencias se encuentran en el presente estudio las actividades físicas practicadas y el tipo de espacio donde practican. Mientras que las mujeres mayores concentran sus actividades físicas semanales en espacios cubiertos, en los varones hay una mayor diversificación y si bien el 80\% también realiza actividades físicas semanales en espacios cubiertos, hay un $20 \%$ que realiza actividad física al aire libre o algún deporte. Además en el presente estudio se ha encontrado que los varones pasean algo más que las mujeres (en lo que debe estar influyendo probablemente un mayor número de mujeres con más edad y con más limitaciones físicas y dependencia funcional, que condicionan junto con el mayor porcentaje de viudas y el vivir solas, que parte de ellas no puedan pasear). Por lo tanto todo parece indicar en este estudio que las mujeres parecen preferir en mayor medida que los varones una práctica de actividad física no deportiva y en espacio cerrado y cubierto, más que en espacio abierto y público.

Con respecto a las actividades y deportes hallados por García Ferrando (2006) en la población general española de 15 a 74 años se observan claras diferencias especialmente con las mujeres mayores de este estudio y algo menores con los varones, pues lo que practican las personas mayores y sobre todo las mujeres son actividades físicas no reglamentadas y muy relacionadas con la salud y la condición física. En ese sentido se observa una gran coincidencia con las actividades identificadas en estudios específicos de mujeres y personas mayores, como el de Graupera et al. (2003), y los estudios de Jiménez-Beatty et al. (2003, 2006) en Madrid y Guadalajara.

Por lo tanto todo parece indicar en el presente estudio (y los estudios precedentes específicos de mujeres y personas mayores) que las mujeres mayores parecen preferir en mayor medida que los varones mayores una práctica en espacio cerrado y cubierto, que en espacio abierto y público. Y este rasgo de una cultura deportiva femenina diferente en la vejez, también parece confirmarse por los datos obtenidos en cuanto a la instalación o espacio donde practican: casi todas las mujeres mayores practican fuera de su casa, bien en un Centro de Mayores o en una Instalación Deportiva o Centro con Instalación Deportiva; sin embargo un tercio de los hombres practica en su casa y un $11 \%$ en lugar público al aire libre (parque, plaza,...) y en mucha menor medida en un Centro de Mayores o en una Instalación Deportiva o Centro con Instalación Deportiva. Estos resultados difieren considerablemente de los obtenidos por García Ferrando (2006) en el conjunto de la población general española de 15 a 74 años, pero coinciden en las tendencias observadas en esta variable, en estudios específicos de mujeres y personas mayores, como el de Graupera et al. (2003), y los estudios de Jiménez-Beatty et al. (2003, 2006) en Madrid y Guadalajara.

En referencia al tiempo de realización semanal de actividad física, las mujeres mayores del presente estudio son unas practicantes muy regulares, casi todas realizan dos horas o más a la semana, con un promedio de 2.53 horas (en los varones mayores sale un promedio una hora superior debido a que un tercio de ellos practican tres horas o más en actividades en la 
Martínez, J.; Jiménez-Beatty J.E.; González, M.D.; Graupera, J.L.; Martín, M.; Campos, A.; Del Hierro, D. (2009). Los hábitos de actividad física de las mujeres mayores en España. Revista Internacional de Ciencias del Deporte. 14(5), 81-93. http://www.cafyd.com/REVISTA/01407.pdf

naturaleza que implican más tiempo, mientras que las mujeres mayores suelen acudir a programas organizados de dos o tres horas a la semana). Estos resultados no son estrictamente comparables con los obtenidos por García Ferrando en 2006, y en los estudios específicos de personas mayores de Graupera et al. y Jiménez-Beatty et al., pues se han medido de manera diferente, pero probablemente podría afirmarse que las mujeres mayores (y los varones mayores) practican como mínimo el mismo tiempo a la semana que los otros grupos sociodemográficos estudiados por García Ferrando y los grupos de mayores estudiados en el municipio de Madrid y en la provincia de Guadalajara.

También parecen observarse diferencias en la localización temporal de la práctica, pues mientras que las mujeres mayores van casi todas (el 75\%) por las mañanas, los varones se distribuyen entre la mañana y la tarde. Este resultado en las mujeres mayores coincide sustancialmente con el obtenido por Graupera en mujeres mayores y por Jiménez-Beatty en las mujeres mayores en el municipio de Madrid, pero difiere con el obtenido en Guadalajara por Jiménez-Beatty, donde las mujeres iban más por las tardes. Estas diferencias entre los resultados de los estudios, quizás vengan propiciadas porque parte de las mujeres y varones mayores a pesar de estar jubilados, no tienen enteramente libre todo el tiempo, sino que mantienen o incorporan una serie de obligaciones y responsabilidades. Y parte quizás venga influido por el horario de oferta o de posibilidad de realización de la actividad. Supuestos que convendría abordar en futuros estudios.

En cuanto al modo de organización de la actividad, se han encontrado notables diferencias: casi todas las mujeres mayores acuden a algún tipo de entidad para realizar su actividad física organizada y la mayoría con profesor, especialmente en Centros de Mayores, seguidos de entidades deportivas municipales, empresas privadas y asociaciones de mayores o de vecinos (apenas asociaciones deportivas); por el contrario la mitad de los hombres mayores autoorganiza su actividad y la otra mitad acude en porcentajes similares a empresas privadas o entidades deportivas municipales y en menor medida a centros de mayores o asociaciones (y además sólo un tercio de los hombres mayores practica con profesor). Estos resultados son bastante similares a los obtenidos en mujeres y varones mayores en el municipio de Madrid y en la provincia de Guadalajara. Sin embargo comparados estos resultados con los datos obtenidos en el conjunto de españoles de 15 a 74 años, hay una cierta aproximación en el caso de los varones mayores pero grandes diferencias en el caso de las mujeres mayores, que como se ha visto en muy escasa medida autoorganizan su actividad, pues recurren casi siempre a algún tipo de entidad y realizan su actividad en grupo y con profesor. Esta tendencia podría deberse a que la cultura deportiva femenina en cuanto a la organización de la actividad coincide en mayor medida con las culturas organizativas y los planteamientos de oferta de clases colectivas en grupos que ofrecen a las personas mayores en primer lugar los propios Centros de Mayores, seguidos de los Ayuntamientos y de las Empresas privadas. Es decir que en el caso de las mujeres mayores podrían estar verificándose también los supuestos avanzados por Puig y Soler (2004) para el conjunto de las mujeres en torno a una mayor adaptación del sector privado comercial y del sector municipal.

En el tiempo de desplazamiento para realizar la actividad no se han encontrado diferencias significativas entre mujeres y varones mayores, empleando la mitad menos de cinco minutos y siendo la media de 8.73 minutos. Pero sí hay diferencias en el modo de desplazamiento, pues mientras que la mayoría de las mujeres mayores acude andando (quizás en parte de ellas porque no tengan vehículo, quizás también porque no tengan carnet de conducir, como consecuencia en gran parte de la muestra de sus roles de madres y amas de casa en sus etapas anteriores del ciclo de vida), en los varones casi la mitad emplea el coche u otros medios 
Martínez, J.; Jiménez-Beatty J.E.; González, M.D.; Graupera, J.L.; Martín, M.; Campos, A.; Del Hierro, D. (2009). Los hábitos de actividad física de las mujeres mayores en España. Revista Internacional de Ciencias del Deporte. 14(5), 81-93. http://www.cafyd.com/REVISTA/01407.pdf

mecánicos de desplazamiento. Estas tendencias de acudir andando, especialmente las mujeres mayores, coinciden ampliamente con los resultados obtenidos en el municipio de Madrid y la provincia de Guadalajara y parecen confirmar una vez más la importancia de la proximidad de las ofertas de actividades a las personas mayores y sobre todo a las mujeres mayores.

\section{Conclusiones}

El porcentaje de mujeres mayores de 65 años en España que realizan actividad física semanal (sin incluir el pasear) es algo superior al de los varones mayores. Si bien éstos parecen pasear algo más que las mujeres mayores. Estas desigualdades vienen acompañadas y quizás explicadas, por una serie de diferencias en la relación de las mujeres mayores con el deporte, o más precisamente con la actividad física, que parecen evidenciar una cultura deportiva femenina en gran parte de las mujeres mayores.

Entre esas diferencias con gran parte de los varones se han observado en las mujeres mayores las siguientes: las actividades físicas semanalmente practicadas (todas a cubierto, ningún deporte y muy relacionadas con el ejercicio físico y la salud); el tipo de instalación o espacio donde practican (casi todas realizan la actividad fuera de su casa y en alguna instalación deportiva o centro que cuente con ella, en espacios cubiertos y cerrados, como salas y vasos de piscinas); la mayoría realiza una actividad física colectiva y organizada por alguna entidad municipal o privada de servicios, con presencia de profesor, dos o tres horas semanales; la mayoría acude andando y emplea menos de diez minutos.

\section{Notas}

${ }^{1}$ La investigación aquí presentada ha formado parte del Proyecto Coordinado de I+D+I DEP2005-00161-C03 que ha sido cofinanciado por el Ministerio de Educación y Ciencia y los fondos europeos FEDER, así como por la Ayuda para I+D de los Grupos de Investigación de la Universidad Politécnica de Madrid: UPM05-C-11203 y la Ayuda para proyectos de investigación de la Universidad de Alcalá.

\section{Referencias bibliográficas}

García Ferrando, M. (2001). Los españoles y el deporte: prácticas y comportamientos en la última década del siglo XX. Madrid: MECD - CSD.

García Ferrando, M. (2006). Posmodernidad y Deporte: Entre la individualización y la masificación. Encuesta sobre hábitos deportivos de los españoles 2005. Madrid: CSD-CIS.

Graupera Sanz, J.L.; Martínez del Castillo, J. y Martín Novo, B. (2003). Factores motivacionales, actitudes y hábitos de práctica de actividad física en las mujeres mayores. Serie ICD de Investigación en Ciencias del Deporte, 35, 181-222.

Jiménez-Beatty, J.E., Graupera Sanz, J.L. y Martínez del Castillo, J. (2003). Hábitos y demandas deportivas de las mujeres mayores en el municipio de Madrid. Serie ICD de Investigación en Ciencias del Deporte, 35, 223-253.

Jiménez-Beatty, J.E.; Martínez del Castillo, J. y Graupera Sanz, J.L. (2006). Las Administraciones públicas de la Provincia de Guadalajara y la integración social de las personas mayores a través de los servicios sociales de actividad físico-deportiva. Alcalá de Henares: Universidad de Alcalá-I lbercaja. 
Martínez, J.; Jiménez-Beatty J.E.; González, M.D.; Graupera, J.L.; Martín, M.; Campos, A.; Del Hierro, D. (2009). Los hábitos de actividad física de las mujeres mayores en España. Revista Internacional de Ciencias del Deporte. 14(5), 81-93. http://www.cafyd.com/REVISTA/01407.pdf

Jiménez-Beatty, J.E.; Graupera Sanz, J.L.; Martínez del Castillo, J.; Campos Izquierdo, A. y Martín Rodríguez, Mạ. (2007). Motivational Factors and Physician Advice for Physical Activity in Older Urban Adults. Journal of Aging and Physical Activity, 15, 236-252.

Martín, M. (1999). Making sense of the first stage in the history of women's rugby in England (1978-1985): difference and multiplicity in gender and rugby. London: Roehampton Institute.

Martínez del Castillo, J.; Graupera Sanz, J.L.; Jiménez-Beatty, J.E.; Rodríguez, M.L. (2006). Condiciones de vida, socialización y actividad física en la vejez. Revista Internacional de Sociología, 44, 39-62.

Mosquera, M.J . y Puig, N. (2002). Género y edad en el deporte. En M. García Ferrando, N. Puig, F. Lagardera (Comps.). Sociología del Deporte (114-141). Madrid: Alianza Editorial.

Puig, N. y Soler, S. (2004). Mujer y deporte en España: estado de la cuestión y propuesta interpretativa. Apunts Educación Física y Deportes, 76, 71-78.

Vázquez Gómez, B. (1993). Actitudes y prácticas deportivas de las mujeres españolas. Madrid: Instituto de la Mujer.

Vázquez Gómez, B. (comp.) (2002). Mujeres y actividades físico-deportivas (Investigaciones en Ciencias del Deporte, no 35). Madrid: Consejo Superior de Deportes. 